\title{
Models of class II methanol masers
}

Andrej M. Sobolev, Andrei B. Ostrovskii, Alexey V. Malyshev, Ural State University, Lenin str. 51, 620083 Ekaterinburg, Russia

Dinah M. Cragg, Peter D. Godfrey, Monash University, Clayton, Victoria 3800, Australia

Edmund C. Sutton, William D. Watson, University of Illinois, 1002 W.Green St., Urbana, IL 61801, USA

Simon P. Ellingsen,

University of Tasmania, Hobart, Tasmania 7001, Australia

Jim L. Caswell

CSIRO, Epping, NSW 2121, Australia

\begin{abstract}
Current models of class II methanol masers are able to describe the brightnesses of the strongest masers and provide a basis for explaining observed line ratios. Determination of the physical parameters in the source requires observational data in many maser transitions. In order to provide observational constraints for models we searched for and detected 7 new methanol masers. This allowed us to constrain the physical parameters of the 3 sources with the greatest number of detected methanol maser lines: W3 $(\mathrm{OH}), \mathrm{NGC6334F}$, and G345.01+1.79. The models accurately account for the fluxes of the bulk of the detected maser lines. Remaining discrepancies most probably reflect the fact that the most prominent components of the different maser lines are formed under different conditions. This is supported by comparison of the line profiles. We outline directions for future studies in the field.
\end{abstract}

Information on methanol maser pumping was first reviewed by Menten (1991), who pointed out that class II methanol masers have radiation as a pumping source. First calculations of excitation (Cragg et al., 1992; Peng \& Whiteoak; 1993, Sobolev, 1993) revealed that these masers arise when the temperature of the pumping radiation exceeds that of masing gas. However, these calculations did not reproduce observed maser intensities.

In order to explain the extremely high brightnesses $\left(>10^{10} \mathrm{~K}\right)$ of the $12 \mathrm{GHz}$ masers, Wilson et al. (1985) supposed that the pumping goes through levels of the 1st torsionally excited state $\left(v_{t}=1\right)$, but calculations of Zeng (1992) failed to reproduce brightnesses this high. The breakthrough was provided by Sobolev \& Deguchi (1994a), who discovered the importance of levels of the 2nd torsionally 
excited state $\left(v_{t}=2\right)$ in the pumping and explained the high brightness of the $12 \mathrm{GHz}$ line. Sobolev \& Deguchi (1994b) subsequently showed that the class II masers are pumped through a great number of cycles. The bulk of the pumping cycles include excitation to the $v_{t}=2$ levels with subsequent decay via $v_{t}=1$. The levels with $v_{t}>0$ play the role of transmitters in the pumping process and need not have large populations. Yet observations of NGC6334F revealed numerous $v_{t}=2$ lines (McCutcheon et al., 2000), and possible $v_{t}=1$ masers were seen in $\mathrm{W} 3(\mathrm{OH})$ by Menten et al. (1986) and Austin, Voronkov \& Sobolev (2000). Strong radiation at about $20 \mu$ is required for efficient pumping (Ostrovskii \& Sobolev, 2001). The presence of dust with considerable optical depth in that range was established by De Buizer, Pina \& Telesco (2000).

The $12 \mathrm{GHz}$ maser spots are small (Menten, 1991). Assuming that the maser sources are of the same size as the spots, Sobolev \& Deguchi (1994a) derived densities $>3 \cdot 10^{6} \mathrm{~cm}^{-3}$ and gas temperatures $<50 \mathrm{~K}$. However, investigations of maser line formation in a turbulent medium have shown that velocity correlations are able to produce a clumpy appearance even for a uniform volume of masing gas (Sobolev, Wallin \& Watson, 1998). In this case the sizes of maser spots reflect the degree of maser amplification and the characteristics of turbulent velocity field, rather than the sizes of actual clumps. Amplification factors for the bright methanol masers exceed $10^{6}$ (the ratio of the maser brightness to that of the background). So, the only constraint on the extent of the maser formation region and the corresponding density might be deduced from the overall spread of maser spots and guesses about the source geometry.

In a study of the brightest methanol masers at $6 \mathrm{GHz}$, Sobolev, Cragg \& Godfrey (1997a) have shown that pumping through $v_{t}=2$ levels is efficient for any density below $10^{8} \mathrm{~cm}^{-3}$, at which density the masers are quenched. Elimination of a lower constraint on density corresponds to a greater freedom in the gas temperature because the latter influences pumping only through collisions. Sobolev, Cragg \& Godfrey (1997a) drew attention to the influence of the bright ultracompact HII regions (UCHII) generally seen near class II methanol maser sources. Although bright masers could be obtained in models without bright backgrounds, seed photons from the UCHII greatly enhance the brightnesses of the low frequency masers. At the same time, the presence of the UCHII greatly increases the number of photons entering the maser region, resulting in saturation of the strong masers. Being stronger at lower frequencies, UCHII emission most affects the $6 \mathrm{GHz}$ masers. As a result the strong sources with the flux ratio $(6 \mathrm{GHz}) /(12 \mathrm{GHz})>15$ are not likely to have an UCHII in the near vicinity. The maser beaming and the gas density also influence the relative brightness of the two strongest transitions, lower beaming and higher density being preferable for sources with low $(6 \mathrm{GHz}) /(12 \mathrm{GHz})$ flux ratios.

Sobolev, Cragg \& Godfrey (1997b) considered the whole set of class II methanol masers produced by pumping by warm dust through levels of the two lowest torsionally excited states. They found that all class II masers detected at that time appear strongly in certain model regimes. An important finding was that various masers react differently to changes in physical parameters. Thus, it is possible to determine maser source parameters through modeling of observational data for many transitions. The paper provided the first list of class II maser candidates based on model calculations. 
Observations of these maser candidates brought detections of new masers at $85.5,86.6,86.9,104.0,165.05,165.06$, and $216.9 \mathrm{GHz}$ and provided tight upper limits for a number of candidates (Sobolev et al., 1999, Cragg et al., 2001, Sutton et al., 2001, Ellingsen et al., in preparation, Sobolev et al., in preparation). It is noteworthy that some of these masers belong to new series of maser transitions and, hence, could not be predicted by extrapolating known series to different J numbers (see, e.g., Menten, 1991 and Sobolev, 1993). To justify the maser nature of this emission in G345.01+1.79, the investigators used the considerable difference in velocities of maser and "thermal" emission. The high brightness temperatures of masers at 86.6 and $86.9 \mathrm{GHz}$ in $\mathrm{W} 3(\mathrm{OH})$ were confirmed by interferometry observations at BIMA (Sutton et al., 2001).

Cragg et al. (2001) performed multitransitional studies of masers in NGC $6334 \mathrm{~F}$ and G345.01+1.79, including the new detections. They constrained the gas density and column density of methanol and attributed differences between maser spectra of these sources to their geometries.

Sutton et al. (2001) constrained the gas temperature and density as well as the methanol column density in the region of formation of a maser spike at -43.1 $\mathrm{km} / \mathrm{s}$ in $\mathrm{W} 3(\mathrm{OH})$. Interestingly, the gas temperature in this region was found to be rather high $(>110 \mathrm{~K})$ in contrast to conditions preferred by the brightest masers. Analysis of data on the broad emission component in the spectra of 9 observed maser candidates have shown that models with "quasithermal" excitation fail to explain their intensities. At the same time, a combination of narrow and broad emission could be well reproduced in a model containing an ensemble of clumps with different elongations and orientations which reside in a shell surrounding the expanding UCHII in $\mathrm{W} 3(\mathrm{OH})$.

Studies of various maser sources show densities in the range of $10^{6}-10^{7} \mathrm{~cm}^{-3}$ and methanol column densities of the order of $5 \cdot 10^{17} \mathrm{~cm}^{-2}$ which imply relative abundances of methanol to hydrogen of about $10^{-6}$. For efficient pumping the dust temperature should exceed $150 \mathrm{~K}$, with the gas temperature having lower values. The strongest masers should be substantially beamed.

The models described above provide an understanding of the basics of class II methanol maser phenomena. However, they have several deficiencies. We will mention the most prominent.

Being one-component LVG models, they don't take into account physical and kinematical structure of the source. VLBI observations of Minier (2000) indicate that maser spots are often accompanied by more extended structures which can be explained both by turbulent velocity correlations and structure of associated clumps.

Substantial parameter variations within the source follow from the noticeable differences between profiles of lines with different intensity (see, e.g., Menten, 1991, Sutton et al., 2001) and even between profiles of relatively weak lines with comparable intensity (Sobolev et al., 1999). Simultaneous observations of a number of maser transitions in G345.01+1.79 show that these profile differences cannot be attributed to maser variability. This issue will be elucidated by multicomponent gaussian analysis with subsequent modeling of excitation. Preliminary studies for G345.01+1.79 and G339.88-1.26 show that the whole sets of maser transitions can be represented by a limited number of components. Another deficiency comes from oversimplification of the dust 
opacity model (Ostrovskii \& Sobolev, 2001). Relevant studies should be done. The last deficiency to be mentioned here comes from insufficient knowledge of transition rates. At present radiative rates are known only for transitions between the ground and two lowest torsionally excited states. Rates for transitions including higher torsionally excited and CO stretch states are greatly needed. Better knowledge of collisional rates is also important for methanol maser research. These rates noticeably influence flux ratios of maser lines in high density sources.

Acknowledgments. Russian participants were supported by INTAS grant 97-11451 and federal program "Astronomy". DMC and SPE were supported by Australian Research Council. ECS was supported by NSF grants AST 96-13999 and AST 99-81363.

\section{References}

Austin, M.C., Voronkov, M.A., \& Sobolev, A.M. 2000, AAS, 197, 2905

Cragg, D.M., Sobolev, A.M., Ellingsen, S.P., Caswell, J.L., Godfrey, P.D., Salii, S.V.,\& Dodson, R.G. 2001, MNRAS, 323, 939

Cragg, D.M., Johns, K.P., Godfrey, P.D., \& Brown, R.D. 1992, MNRAS, 259, 203

Cragg, D.M., Sobolev, A.M., Ellingsen, S.P., Caswell, J.L., Godfrey, P.D., Salii, S.V., \& Dodson, R.G. 2001, MNRAS, in press

De Buizer, J.M., Pina, R.K.,\& Telesco, C.M. 2000, ApJS, 130, 437

McCutcheon, W.H., Sandell, G., Matthews, H.E., Kuiper, T.B.H., Sutton, E.C., Danchi, W.C. \& Sato, T. 2000, MNRAS, 316, 152

Menten, K.M. 1991, PASP, 16, 119

Menten, K.M., Walmsley, C.M., Henkel, C., Wilson, T.L., Snyder, L.E., Hollis, J.M., \& Lovas, F. 1986, A\&A, 169, 271

Minier, V. 2000, PhD thesis, Onsala Space Observatory, 75

Ostrovskii, A.B.,\& Sobolev, A.M. 2001, these proceedings

Peng, R.S., \& Whiteoak, J.B. 1993, Lect. Notes Phys., 412, 207

Sobolev, A.M. 1993, Astron. Lett., 19, 293

Sobolev, A.M., Cragg, D.M.,\& Godfrey, P.D. 1997a, A\&A, 324, 211

Sobolev, A.M., Cragg, D.M.,\& Godfrey, P.D. 1997b, MNRAS, 288, L39

Sobolev, A.M., Cragg, D.M., Salii, S.V., Kalinina, N.D., \& Ellingsen, S.P. 1999, Proc. 3rd Cologne-Zermatt Symposium, GCA-Verlag Herdecke, 299

Sobolev, A.M., \& Deguchi, S. 1994a, A\&A, 291, 569

Sobolev, A.M., \& Deguchi, S. 1994b, ApJ, 433, 719

Sobolev, A.M., Wallin, B.K., \& Watson, W.D. 1998, ApJ, 498, 763

Sutton, E.C., Sobolev, A.M., Ellingsen, S.P., Cragg, D.M., Mehringer, D.M., Ostrovskii, A.B., \& Godfrey, P.D. 2001, ApJ, in press

Wilson, T.L., Walmsley, C.M., Menten, K.M., \& Hermsen, W. 1985, A\&A, 147, L19

Zeng, Q., 1992, IAU Coll. 143, 341 